\title{
BUBBLE MEASUREMENTS DOWNSTREAM OF HYDRAULIC JUMPS
}

\author{
Tricia A. Waniewski \\ Christopher Hunter \\ Christopher E. Brennen
}

\begin{abstract}
A phase Doppler anemometer (PDA) system was used to measure the velocity profiles and air bubble size distributions downstream of two-dimensional hydraulic jumps for different upstream flow conditions in a $1.92 \mathrm{~m}$ long laboratory flume. The PDA detected bubbles from 1 to $500 \mu \mathrm{m}$ in diameter, and more were found at the bottom of the downstream flow near the elevation of the upstream free surface. This distribution was more marked for smaller bubbles, those with diameters less than $100 \mu \mathrm{m}$. The migration of the bubbles is controlled by the effects of buoyancy and by turbulent mixing; the relative magnitude of their effects on bubble distribution depends strongly on bubble size, and also on the energy of the upstream flow.
\end{abstract}

\section{Introduction}

Though hydraulic jumps are commonly occurring flow phenomenon, they are also very complex. A hydraulic jump normally involves not only an abrupt change in depth, a significant loss of energy, and the production of turbulence, but also the entrainment of air which is the focus of the present paper. An early study of air entrainment in hydraulic jumps by Resch et al. (1974) measured void fractions and bubble size distributions for different inflow conditions using hot film anemometry techniques. Babb and Aus (1981) made similar measurements, enhancing the hot film anemometry techniques with gamma-ray attenuation. 
Hoyt and Sellin (1989) used drag-reducing additives in their experimental studies to observe the air entrainment process. They likened the hydraulic jump to a mixing layer and used expressions from gas mixing layers to estimate the quantity of air entrained. Chanson (1995) summarizes these and other studies, and compares them to his void fraction measurements using a conductivity probe. More recently, Mossa and Tolve (1998) developed a flow visualization technique which allowed them to examine flow structures such as the vortices in the surface roller and to estimate the air concentration in the flow. These studies have led to empirical correlations which predict oxygen transfer; however, they generally focus on the flow in the immediate vicinity of the hydraulic jump and do not examine the evolution of the air bubble populations in the flow further downstream. Understanding the evolution of these downstream bubble populations is important; for example, bubbles entrained by similar processes in ship bow waves can persist in the wake for distances large enough to affect the ship's radar signature. The evolution of these bubble populations depends quite critically on the downstream turbulent flow field.

The turbulence characteristics of hydraulic jumps also has been an active area of research. Rouse et al. (1958) conducted some of the first research in this area, exploring the turbulence characteristics using hot wire measurements in an air flow model of a hydraulic jump. Rajaratnam (1965) measured velocity profiles in hydraulic jumps and likened them to those in a "wall jet". McCorquodale and Khalifa (1983) propose a hydraulic jump model which, among other things, can predict the velocity distribution and water surface elevation. They included the effects of air entrainment, turbulent shear, and turbulence pressure whose effects increased with the square of the upstream Froude number, $\mathbf{F}_{1}^{2}$. Qingchao and Drewes (1994) calculated the turbulence characteristics for free and forced hydraulic jumps using the $K$-epsilon model adapted for a moving free surface. Though their model did not take multi-phase flows effects into account, their calculations showed good agreement with experimental data. Wu and Rajaratnam (1996) consider the transition from hydraulic jump to open channel flow. 


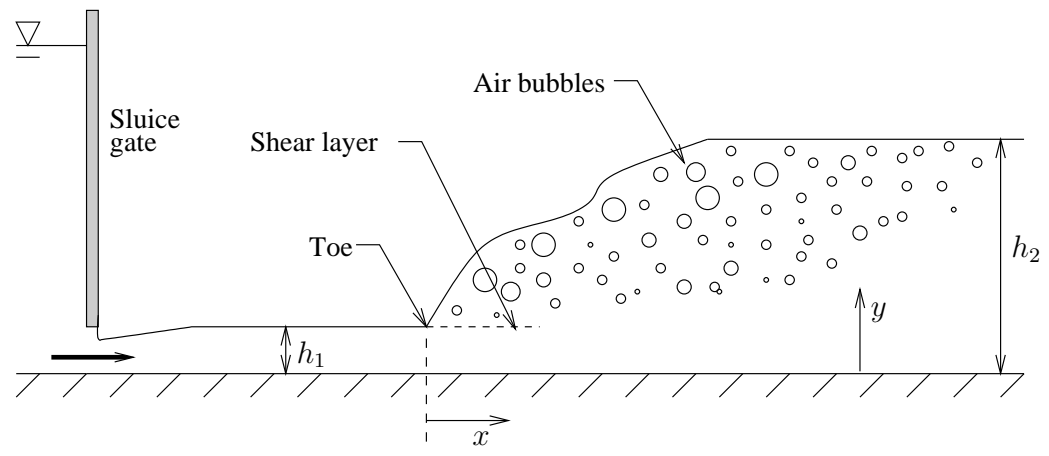

Figure 1: Schematic diagram of a hydraulic jump in the flume (not to scale). The measurement locations were approximately $1 \mathrm{~m}$ downstream of the toe of the hydraulic jump.

The present paper examines the bubble size distributions downstream of a hydraulic jump created in a laboratory flume and considers the relative influence of bubble buoyancy and turbulence in determining these distributions. In doing so we also examine the mean velocity distributions and the fluctuating or turbulent velocities.

\section{Experimental apparatus}

The experiments were performed in a recirculating $1.92 \mathrm{~m}$ long horizontal laboratory flume shown schematically in Figure 1. The flume was $15.2 \mathrm{~cm}$ wide and $20.3 \mathrm{~cm}$ deep with glass sidewalls and a galvanized aluminum bottom. A glass window $(17.8 \times 7.6 \mathrm{~cm})$ was installed in the bottom, its center $1.06 \mathrm{~m}$ from the sluice gate at the upstream end. The flume was filled with fresh water which was filtered using a $5 \mu \mathrm{m}$ polypropylene filter for several hours before the experiments. The flow rate, $Q$, was measured using a Venturi meter and the depth with a point gage. A supercritical flow was generated using an adjustable vertical sluice gate to control the discharge from an upstream reservoir. The height of the opening was varied between approximately 3 and $7 \mathrm{~mm}$ in the experiments, yielding values of $\mathbf{F}_{1}$ from 5 to 20. The hydraulic jumps were created by adjusting the vertical position of a weir at the downstream end of the flume. The distance from the vertical sluice gate to the toe of 
the hydraulic jump was about $1 \mathrm{~cm}$.

Resch et al. (1974) demonstrated that the upstream flow conditions can affect the characteristics of hydraulic jumps including the air entrainment. In the present experiments, the location of the hydraulic jump toe was within a few centimeters of the sluice gate where the local Reynolds number is on the order of $10^{3}$. The boundary layer thickness on the bottom of the flume may be estimated using $\delta / x \approx 5 / R e_{x}^{1 / 2}$ where $\delta$ is the boundary layer thickness, $x$ is the distance from the location of the sluice gate, and $R e_{x}$ is the local Reynolds number (White, 1986). Then for an upstream velocity $U_{1}$ of $2.78 \mathrm{~m} / \mathrm{s}$ and a distance $x$ of $1 \mathrm{~cm}, \delta$ is $0.3 \mathrm{~mm}$. The upstream depth, $h_{1}$, for this flow was $6 \mathrm{~mm}$; therefore, $\delta / h_{1}=0.05$. Consequently, in the present experiments the inflow boundary layer thickness was small compared with the depth and the inflow may be considered as partially developed.

A phase Doppler anemometer (PDA) system from Dantec Measurement Technology deployed in a forward scattering configuration with a scattering angle of $68^{\circ}$ was used to measure the velocity and the diameter, $d$, of the air bubbles entrained by the hydraulic jump. The transmitting and receiving optics were mounted on separate positioning systems so that the entire depth of a streamwise section of the flow approximately $10 \mathrm{~cm}$ long could be observed. The measurement volume of the PDA was located on the centerline of the flume, about $1 \mathrm{~m}$ downstream of the toe of the hydraulic jump. An optical mirror mounted at a $34^{\circ}$ angle to the ground beneath the glass window in the bottom of the flume directed the scattered light from particles passing through the PDA measurement volume into the receiving optics which included three photomultiplier tubes (PMTs). Dantec Measurement Technology reports that for most configurations of the PDA system, the velocity measurements are accurate to within 1\% (Dantec, 1991). For the present experiments, the PDA was configured to measure bubbles with diameters less than $517 \mu \mathrm{m}$ with a resolution of $1 \mu \mathrm{m}$ (9 bits). Therefore, bubbles smaller than $1 \mu \mathrm{m}$ were not detected. Dantec Measurement Technology reports the diameter measurements are accurate to within 4\% (Dantec, 1991).

The signals from the PMTs were partially processed by the PDA signal processor and 
the information was stored on the hard disk of a 486 PC. Particles causing a phase error of greater than $20^{\circ}$ were rejected as non-spherical samples; these were probably dirt particles and not air bubbles. The processing was completed at a later time using "SIZEware", the software provided by Dantec Measurement Technology. The result is a count rate, $C(d)$, which is the number of bubbles passing through the measurement volume per second. The corresponding bubble number density, $\rho_{N}$, was calculated using

$$
\rho_{N}(d)=\frac{C(d)}{u(d) A(d, \ldots)}
$$

where $u$ is the bubble velocity and $A$ is the cross-sectional area of the measurement volume. The optical, particle, and electronic parameters all interact to determine this cross-sectional area as described by Saffman (1987); however, the following simplified calculation method was used for the present paper. The diameter of the focused beam, $\delta_{f}$, was assumed to be constant and was calculated using

$$
\delta_{f}=\frac{4 f \lambda}{\pi E \delta_{l}}
$$

where $f$ is the focal length of the front lens, $\lambda$ is the laser wavelength, $E$ is the beam expansion factor, and $\delta_{l}$ is the diameter of the beam waist before expansion (Dantec, 1991). The crosssectional area is the fraction of the measurement volume visible to the receiving optics, and this was approximated by the intersection of the projection of the spatial filter (slit) in the receiving optics and the focused beam. Using this method for the present experimental configuration, $A$ was estimated to be $0.0356 \mathrm{~mm}^{2}$. Note that this is only an approximation; due to this uncertainty the bubble count rates rather than bubble number distributions will be presented though the latter could be estimated using equation 1.

\section{Phase Doppler anemometer validation}

A preliminary set of experiments were performed to validate the bubble diameters reported in the PDA bubble size distribution measurements. The PDA bubble size measurements were 
calibrated in a different facility at an earlier date using measurements of bubble diameters from holographic reconstruction of the same sample volume. Details of this calibration procedure are given in Liu et al. (1993). In the present validation experiments, the flume was used to generate a subcritical flow $(U=0.141 \mathrm{~m} / \mathrm{s}, h=9.37 \mathrm{~cm}$, and $\mathbf{F}=0.15)$ in which air bubbles having a known size distribution were introduced. The air bubbles were produced using a diaphragm aquarium pump to force air through a porous glass frit 12.6 $\mathrm{cm}$ by $2.9 \mathrm{~cm}$ by $0.5 \mathrm{~cm}$ thick, glued into a plastic trough. The trough was mounted on the bottom of the flume approximately $6 \mathrm{~cm}$ upstream of the PDA measurement volume. Skop et al. (1989) measured the probability density distribution for bubbles produced by forcing air at 100-300 $\mathrm{mL} / \mathrm{min}$ though this same frit. In their experiments, the frit was mounted near the base of a lucite tank filled with artificial seawater which was $15.8 \mathrm{~cm}$ long, 10.0 $\mathrm{cm}$ wide, and $32.5 \mathrm{~cm}$ high. The water in the tank was agitated by a magnetic stirring rod located on the base. Bubble diameters were measured from enlarged photographs of 60 bubbles in the tank; the mean diameter was $308 \mu \mathrm{m}$ with a standard deviation of $87 \mu \mathrm{m}$ and the measured distribution appeared to be lognormal.

Typical results from the present validation experiments are shown in Figure 2. The bubbles were grouped into 50 bins according to size, the bin size being $10.3 \mu \mathrm{m}$. For example, the count rate for bubbles between $82.7 \mu \mathrm{m}$ and $93.0 \mu \mathrm{m}$ in diameter is represented by a single point at $87.9 \mu \mathrm{m}$ on the abscissa. The sampling time in these experiments was 180 seconds. The large peaks at $15-70 \mu \mathrm{m}$ in Figure 2 clearly are due to the mean background bubble distribution present in the flume. This background bubble population probably results from the air entrainment by the free jet from the weir falling into the reservoir. On the other hand, the peak around $300 \mu \mathrm{m}$ is clearly due to the air injection by the frit. The bubble size distributions measured by Skop et al. (1989) for this same frit had a mean of $308 \mu \mathrm{m}$; however, only a rough comparison can be made since the two experiments had different air flow rates through the frit, different velocities over the frit, and were conducted with different salinities of water. 


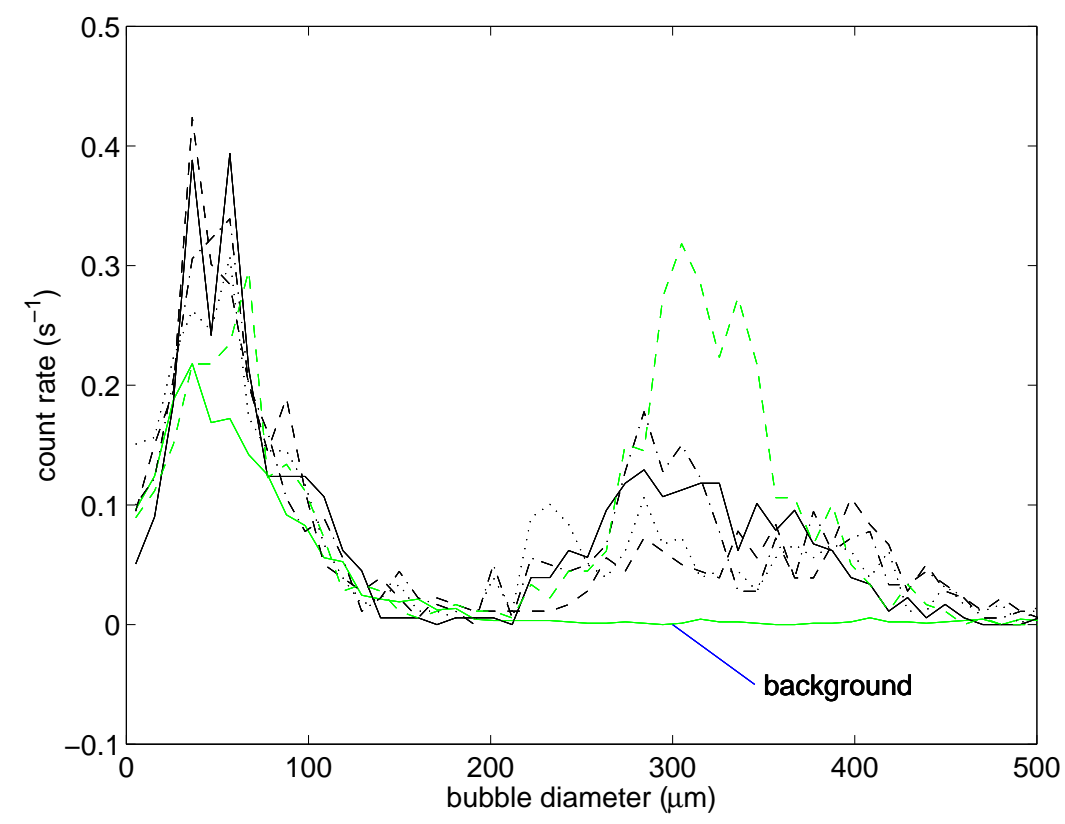

Figure 2: Five repeated bubble size distribution measurements at $y=2.5 \mathrm{~cm}$ for bubbles introduced by a frit in a subcritical flow where $\mathbf{F}=0.15$. The mean background bubble size distribution is indicated.

\section{Velocity profiles and bubble size distributions}

The main present experiments concentrated on measurement of the bubble size distributions at locations approximately $1 \mathrm{~m}$ downstream of the hydraulic jump. The majority of the bubbles at this location were produced by the hydraulic jump; however, it is possible that a few smaller bubbles were present in the inflow upstream of the hydraulic jump. These inflow bubbles originated either from the hydraulic jump or from the free jet from the weir falling into the reservoir and were recirculated through the flume. However, considering the background bubble distribution shown in Figure 2 it is estimated that the number of inflow bubbles was at least an order of magnitude less than the number of bubbles produced by the hydraulic jump.

Initial experiments studied the change in the bubble size distribution with time for the same location $(x=1 \mathrm{~m}$ and $y=1.27 \mathrm{~cm})$, and flow conditions $\left(\mathbf{F}_{1}=17.3\right)$. The sampling time 


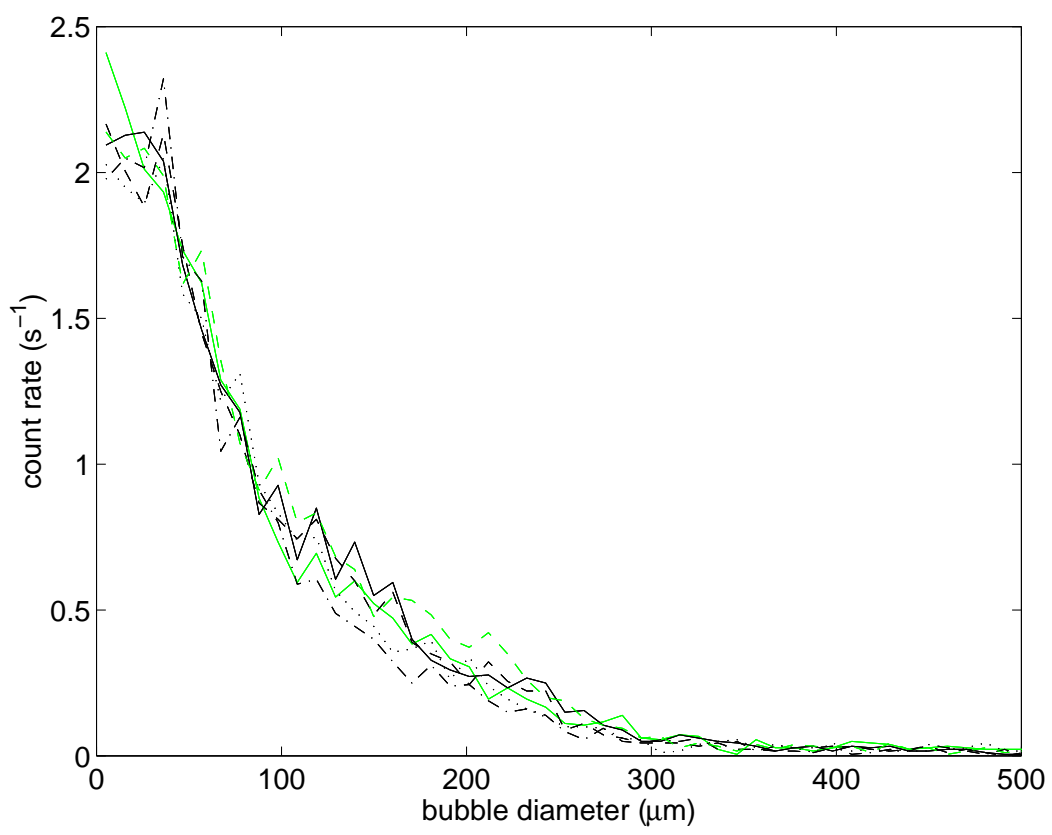

Figure 3: Six bubble size distribution measurements downstream from a hydraulic jump at $x=1 \mathrm{~m}, y=0.0127 \mathrm{~m}$ with an upstream Froude number, $\mathbf{F}_{1}$, of 17.3 . About seven minutes lapsed between the beginning of the sampling times for each of these distributions.

was 180 seconds, and samples were taken at elapsed times of 0, 5, 13, 20, 31, and 40 minutes. The average number of spherical bubbles detected for these experiments was 4,066. The bubble size distribution results are shown in Figure 3, and indicate that the measurements are repeatable and that the count rates are accurate to within about $\pm 0.14 \mathrm{~s}^{-1}$. This figure also demonstrates that the bubble population in the facility reaches a steady state in a matter of minutes which contrasts with bubble populations in larger facilities which can have much longer adjustment times (for example, Liu et al. (1993)).

Additional bubble diameter and velocity distributions downstream of the hydraulic jump were measured for the three flow conditions summarized in Table 1. The depths and the flow rate were measured, and the velocities were calculated using the continuity equation. The continuity and momentum equations for the hydraulic jump yield the well known relation 


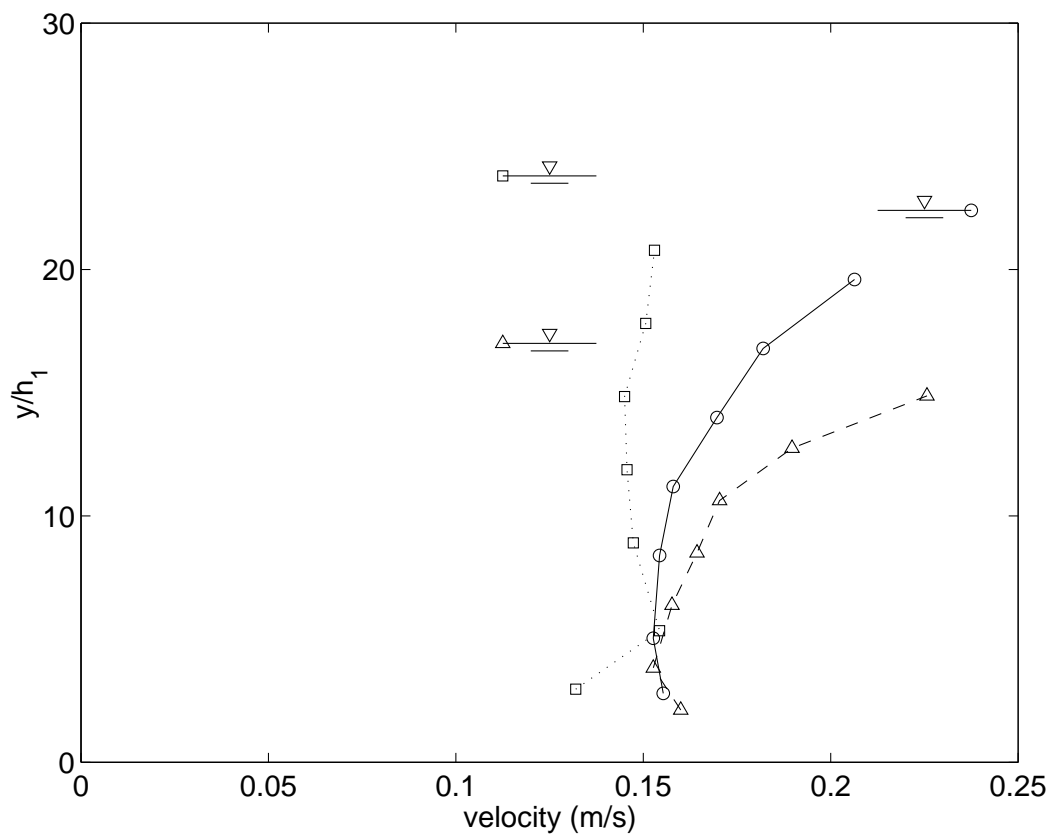

Figure 4: Velocity, $\overline{u_{2}}$, profiles downstream of the hydraulic jump; (- -) for $\mathbf{F}_{1}=11.5,(-)$ for $\mathbf{F}_{1}=17.1$, and $(\cdots)$ for $\mathbf{F}_{1}=19.3$. The mean location of the free surface also is indicated for each flow condition. 


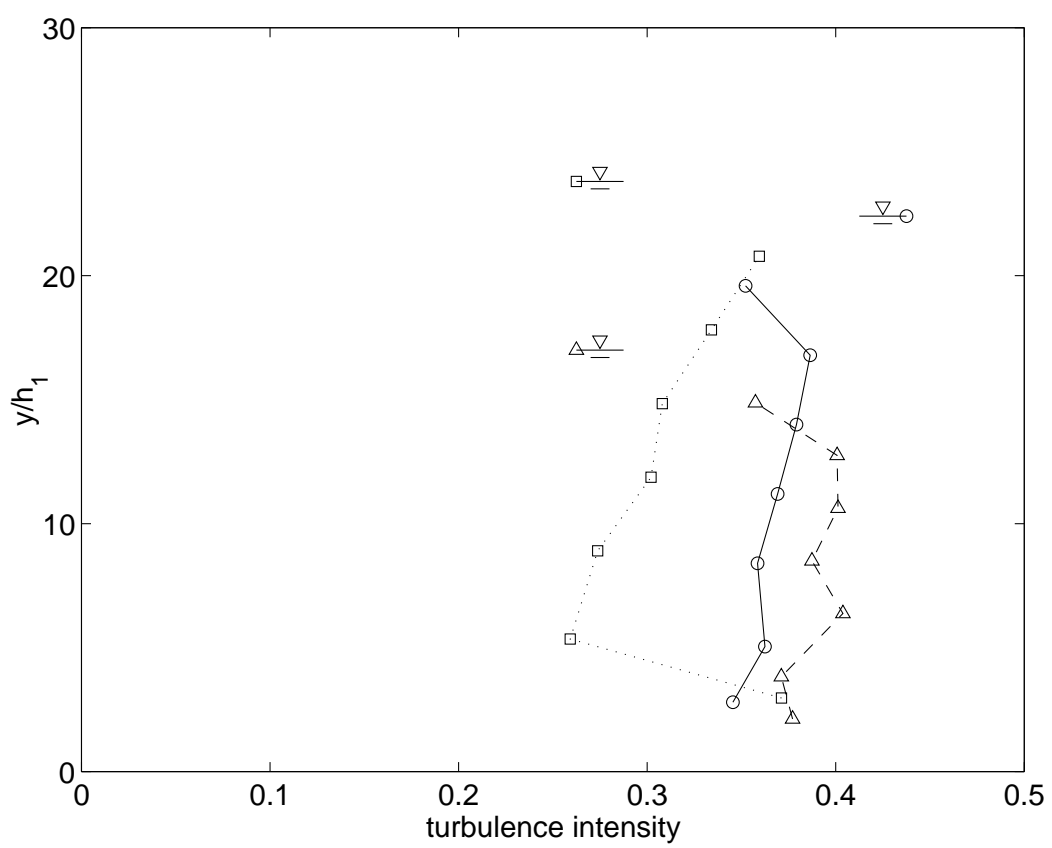

Figure 5: Turbulence intensity profiles downstream of the hydraulic jump; (- -) for $\mathbf{F}_{1}=$ 11.5, (-) for $\mathbf{F}_{1}=17.1$, and $(\cdots)$ for $\mathbf{F}_{1}=19.3$. The mean location of the free surface also is indicated for each flow condition. 


\begin{tabular}{|c|c|c|c|c|c|}
\hline Flow & $\begin{array}{c}h_{1} \\
(\mathrm{~cm})\end{array}$ & $\begin{array}{c}U_{1} \\
(\mathrm{~m} / \mathrm{s})\end{array}$ & $\mathbf{F}_{1}$ & $\begin{array}{c}h_{2} \\
(\mathrm{~cm})\end{array}$ & $\begin{array}{c}U_{2} \\
(\mathrm{~m} / \mathrm{s})\end{array}$ \\
\hline \hline 1 & 0.60 & 2.78 & 11.5 & 10.2 & 0.164 \\
2 & 0.46 & 3.61 & 17.1 & 10.3 & 0.161 \\
3 & 0.32 & 3.42 & 19.3 & 7.6 & 0.144 \\
\hline
\end{tabular}

Table 1: Summary of experimental flow conditions for the hydraulic jump experiments.

between the upstream and downstream depths,

$$
\frac{h_{2}}{h_{1}}=-\frac{1}{2}+\sqrt{\frac{1}{4}+2 \mathbf{F}_{1}{ }^{2}} .
$$

Using equation 3 , the calculated values of $h_{2} / h_{1}$ agreed with the measured values to within $\pm 10 \%$. These discrepancies were probably due to the experimental error in measuring $h_{2}$; downstream of the hydraulic jump the free surface fluctuations were about $\pm 1 \mathrm{~cm}$.

Measurements were performed at seven elevations for three different streamwise locations: $x=0.987 \mathrm{~m}, x=1.000 \mathrm{~m}$, and $x=1.013 \mathrm{~m}$. However, since they are essentially the same, the data was averaged over these three streamwise locations. The velocity profiles for the three flow conditions are shown in Figure 4. The shape of these profiles are similar to those measured by Wu and Rajaratnam (1996) for an upstream Froude number, $\mathbf{F}_{1}$, of 10.48. The turbulence intensity, $T$, was calculated using

$$
T=\frac{\sqrt{\overline{u_{2}^{\prime}}}}{\overline{u_{2}}}
$$

where $u_{2}^{\prime}$ is the fluctuating velocity component and $\overline{u_{2}}$ is the mean velocity at locations downstream of the hydraulic jump. Figure 5 shows the turbulence intensity profiles for the three flow conditions. Wu and Rajaratnam (1996) found the maximum turbulence intensity to vary from 0.28 to 0.38 in their experiments for flows with upstream Froude numbers from 3.87 to 10.48 ; the highest values of turbulence intensity occurred for flows with greatest Froude numbers. This is consistent with the present experiements where the maximum 
turbulence intensity varied from 0.35 to 0.42 for flows with upstream Froude numbers from 11.5 to 19.3; however, in the present experiments the maximum value of turbulence intensity decreased with Froude number. It is possible that this discrepancy is due to differences in the experimental facilities.

The bubble size data is summarized in Figures 6, 7, and 8. For clarity in interpreting these figures, only half of the bubble diameter distributions (every alternate elevation) are shown. In general, the distributions are positively skewed with means of $78-116 \mu \mathrm{m}$ and modes around $40 \mu \mathrm{m}$. They appear similar to Gamma distributions, tapering to zero at 300 $\mu \mathrm{m}$. Figure 9 shows the variation in the mean bubble diameter with depth. In addition, the bubble size distributions were integrated, and the variation in the total bubble count rate with depth is shown in Figure 10 for each flow condition.

\section{Discussion}

In a hydraulic jump, the air entrainment mechanisms are impingement of the jet-like upstream flow on the surface roller at approximately $y=h_{1}$ (Rajaratnam, 1965; Chanson, 1995) and the counter-rotating vortices in the surface roller (Hoyt and Sellin, 1989; Mossa and Tolve, 1998). Detailed descriptions of both air entrainment mechanisms can be found in the literature by these and other researchers. From visual observations of high Froude number $\left(\mathbf{F}_{1}>10\right)$ flows in the present experiments, the impingement of the upstream flow seemed to entrain more air than the counter-rotating vortices. Downstream of the hydraulic jump, the migration of the air bubbles is controlled by the effects of buoyancy and turbulent mixing. The entrained air is present in a broad spectrum of bubble sizes, and the relative effects of buoyancy and turbulent mixing will differ for different bubble sizes for a given flow. The bubble size distributions will evolve with downstream distance and may vary significantly with depth.

Though the following analysis is rather tentative, to examine the relative effects of the 


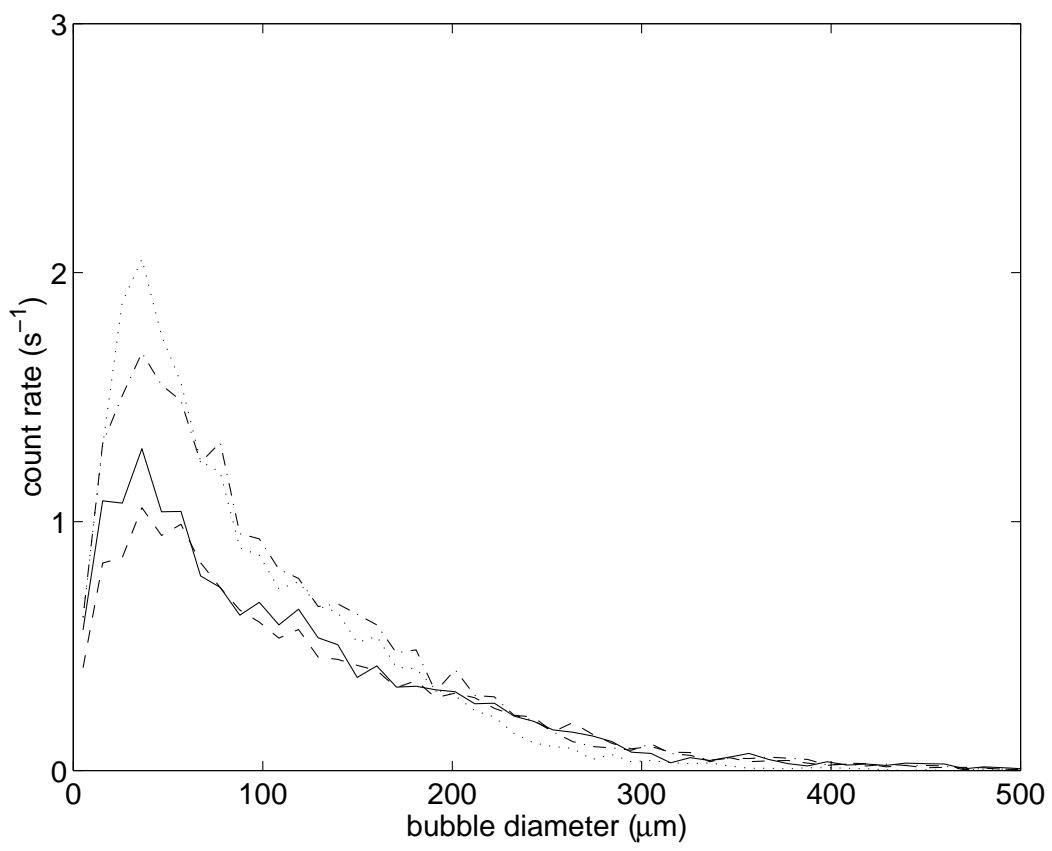

Figure 6: Bubble size distributions downstream of a hydraulic jump with $\mathbf{F}_{1}=11.5$. Each line represents a different elevation: $(\cdots)$ for $y / h_{1}=2.13,(-\cdots)$ for $y / h_{1}=6.38,(--)$ for $y / h_{1}=10.6$, and $(-)$ for $y / h_{1}=14.9$. The mean free surface is located at $y / h_{1}=17.0$. 


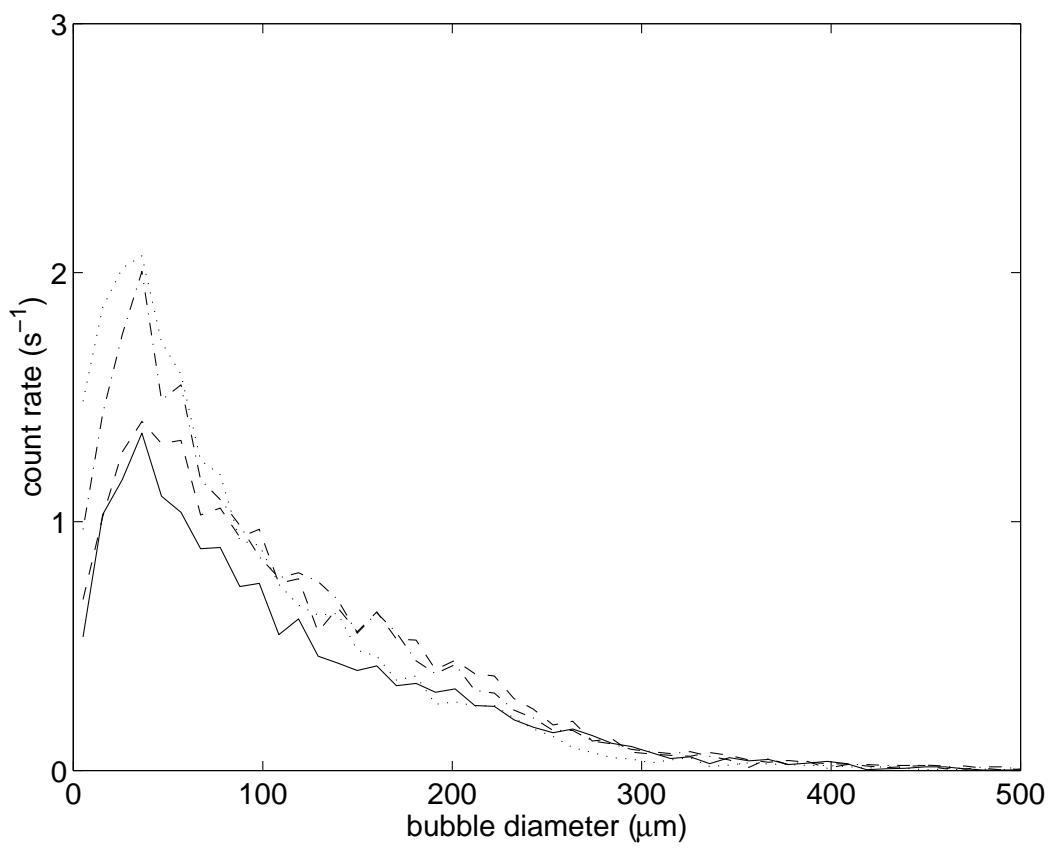

Figure 7: Bubble size distributions downstream of a hydraulic jump with $\mathbf{F}_{1}=17.1$. Each line represents a different elevation: $(\cdots)$ for $y / h_{1}=2.80,(-\cdots)$ for $y / h_{1}=8.40,(-)^{-}$for $y / h_{1}=14.0$, and $(-)$ for $y / h_{1}=19.6$. The mean free surface is located at $y / h_{1}=22.4$. 


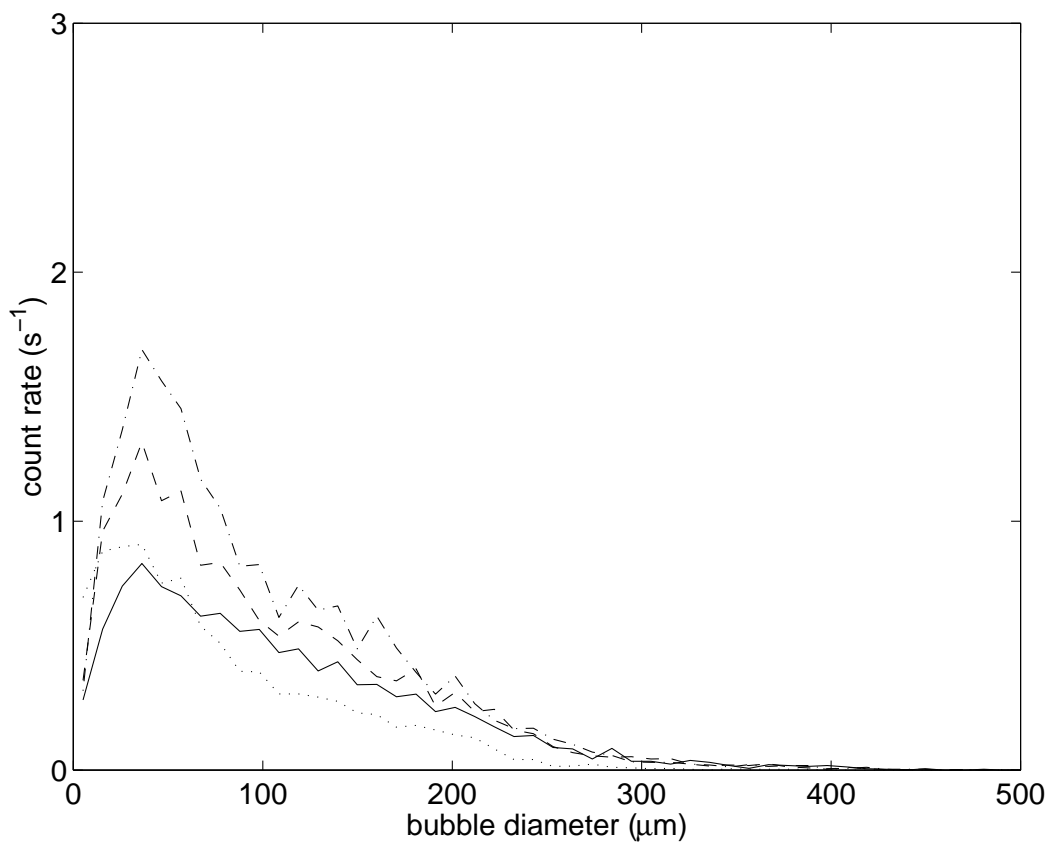

Figure 8: Bubble size distributions downstream of a hydraulic jump with $\mathbf{F}_{1}=19.3$. Each line represents a different elevation: $(\cdots)$ for $y / h_{1}=2.97,(-\cdots)$ for $y / h_{1}=8.91,(--)$ for $y / h_{1}=14.8$, and $(-)$ for $y / h_{1}=20.8$. The mean free surface is located at $y / h_{1}=23.8$.

upward buoyant motion and the turbulent mixing on the bubble population distribution one may construct an equation governing the evolution of the number of bubbles of a particular radius $R, N(x, y, R)$, by starting with the two-dimensional conservation of bubble number

$$
\frac{\partial \bar{N}}{\partial t}+\frac{\partial}{\partial x_{i}}\left(\bar{N} \overline{u_{i}}\right)+\frac{\partial}{\partial x_{i}}\left(\overline{N^{\prime} u_{i}^{\prime}}\right)=0
$$

where the velocity, $u_{i}$, and the density, $\rho$, are instantaneous fluctuating quantities defined as $u_{i}=\overline{u_{i}}+u_{i}^{\prime}$ and $N=\bar{N}+N^{\prime}$. Then, one may assume: (a) isotropic turbulence, (b) a mean vertical velocity equal to the terminal rise velocity, $V(R)$, (c) no creation or destruction of bubbles, and (d) no bubble growth. In addition, one may assume a mixing length, $\ell$, which determines the relation of the fluctuating population, $N^{\prime}$, to the gradient of the mean population, $\bar{N}$, according to

$$
N^{\prime}=\ell \frac{\partial \bar{N}}{\partial y},
$$




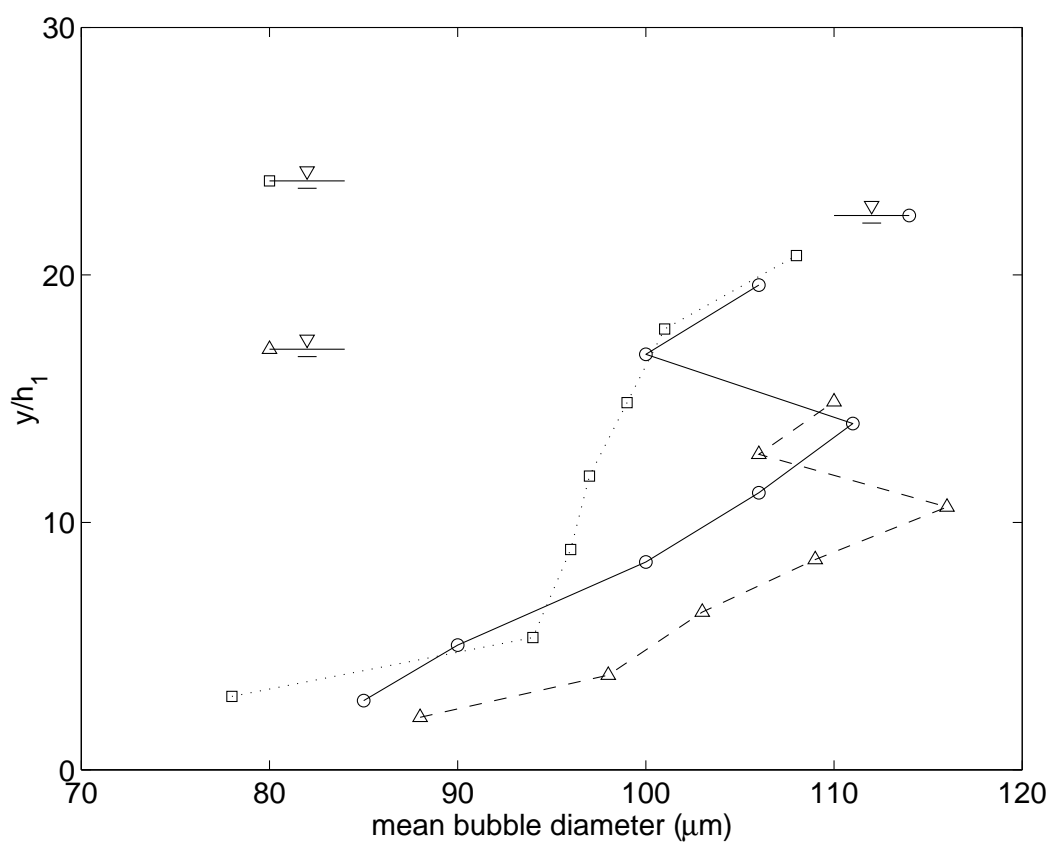

Figure 9: Mean bubble diameter, $\bar{d}$, downstream of a hydraulic jump as a function of elevation, $y / h_{1}$ : (- -) for $\mathbf{F}_{1}=11.5,(-)$ for $\mathbf{F}_{1}=17.1$, and $(\cdots)$ for $\mathbf{F}_{1}=19.3$. The mean location of the free surface also is indicated for each flow condition. 


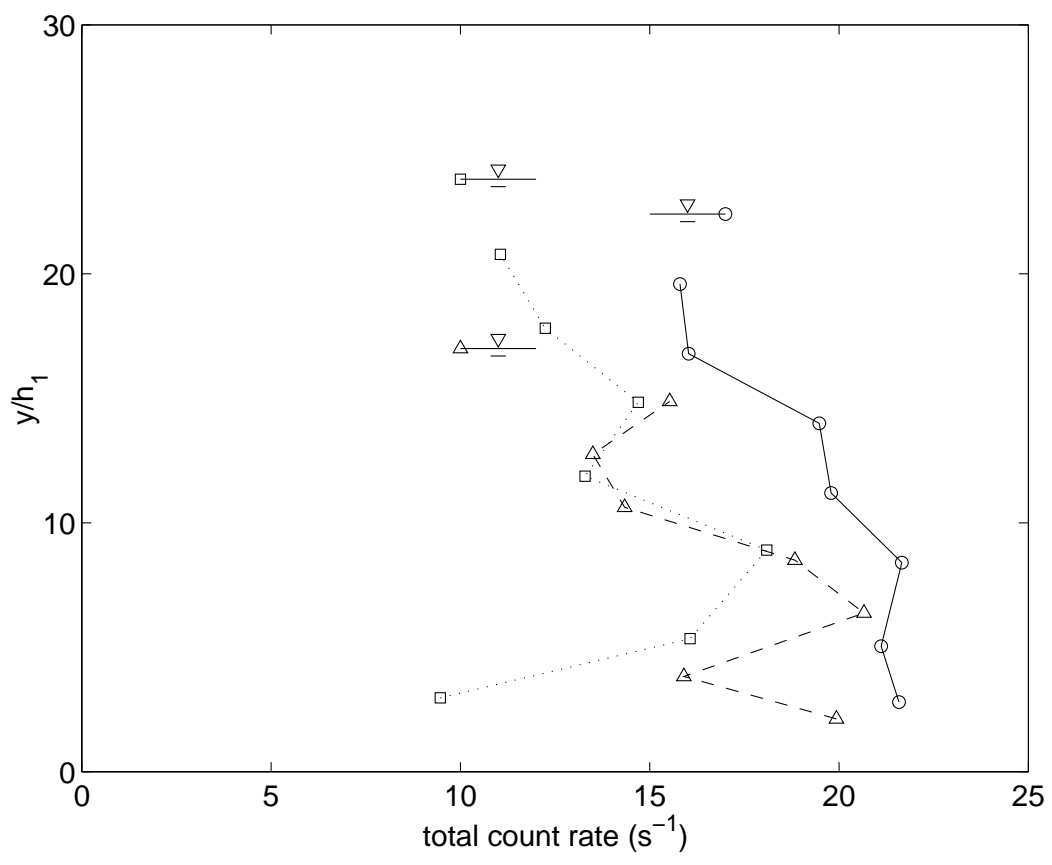

Figure 10: Total bubble count rate downstream of a hydraulic jump as a function of elevation, $y / h_{1}$ : (- $)$ for $\mathbf{F}_{1}=11.5,(-)$ for $\mathbf{F}_{1}=17.1$, and $(\cdots)$ for $\mathbf{F}_{1}=19.3$. The mean location of the free surface also is indicated for each flow condition. 


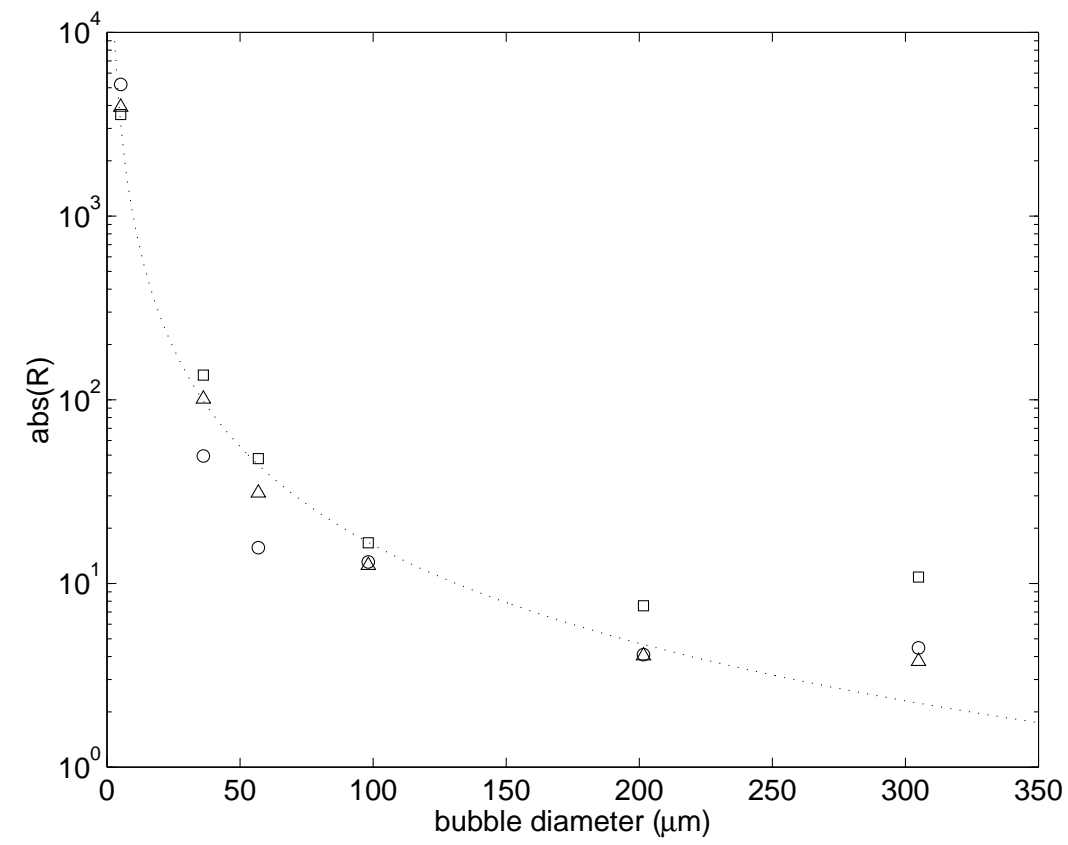

Figure 11: The absolute value of ratio $\mathcal{R}$ as a function of bubble diameter: $(\triangle)$ for $\mathbf{F}_{1}=$ 11.5, (o) for $\mathbf{F}_{1}=17.1$, and $(\square)$ for $\mathbf{F}_{1}=19.3$. A least squares curve fit, $|\mathcal{R}|=58300 d^{-1.78}$, is shown for the $\mathbf{F}_{1}=11.5$ data.

and $\ell$ is estimated using Prandtl's hypothesis,

$$
\ell=\frac{u^{\prime}}{\partial \bar{u} / \partial y}
$$

This construction leads to

$$
\frac{\partial}{\partial x}(\bar{N} \bar{u})=\frac{\partial}{\partial y}\left(-\bar{N} V-\ell u^{\prime} \frac{\partial \bar{N}}{\partial y}\right)
$$

A measure of the relative magnitudes of the buoyancy effect and the turbulent mixing effect can be obtained from the ratio, $\mathcal{R}$, of the two terms inside the $\partial / \partial y$ on the right hand side of equation 8 ,

$$
\mathcal{R}=\frac{\ell u^{\prime} \frac{\partial \bar{N}}{\partial y}}{\bar{N} V} .
$$

This ratio was evaluated using the experimental data. The terminal rise velocities, $V$, were calculated according to the correlations recommended by Wallis (1969). Figure 11 shows 
the absolute value of ratio $\mathcal{R}$ as a function of bubble diameter for different Froude numbers. The turbulence term clearly becomes more important for the smaller bubbles, and this is valuable in understanding some of the trends in the experimental data.

We now examine the flow characteristics and bubble distributions presented in a previous section. Figures 6 and 7 are for flows with almost the same downstream depth and mean velocity (see Table 1); therefore, the bubble number distributions and their variation with depth are very similar. The number of bubbles increases with depth, and this increase is particularly noticeable for the smaller bubbles, those less than about $100 \mu \mathrm{m}$ in diameter. This is not surprising since most of the air is entrained at $y=h_{1}$; however, other depths are substantially populated with both smaller and larger bubbles and we conclude that the effects of turbulent mixing are dominant for these flows. This is also shown in Figure 11 where $|\mathcal{R}|>1$ for all bubble sizes.

In contrast, Figure 8 has a substantially smaller downstream depth and a somewhat smaller downstream velocity; the upstream flow is shallower and less energetic. As a result the total bubble count rate for this flow is less than the total bubble count rate for the other two Froude numbers (Figure 10). Moreover, the variation in the bubble distributions with depth exhibits a notable difference in that there are significantly fewer bubbles near the bottom than at the upper elevations (or near the bottom in Figures 6 and 7). It is likely that this depopulation of the lowest layer of the flow is due to the effects of buoyancy, and since this flow was less energetic the effects of buoyancy are more noticeable. In fact, Figure 9 shows a greater change in mean bubble diameter for the $\mathbf{F}_{1}=19.3$ flow than for the other two flows. However this is not reflected by the values of ratio $\mathcal{R}$ shown in Figure 11; the $\mathcal{R}$ values for the $\mathbf{F}_{1}=19.3$ flows are actually greater than those calculated for the other two flows. This may be because of scatter in the data which is due, in part, to using finite differences to evaluate the $\partial / \partial y$ derivatives.

In summary, while the effects of turbulent mixing are certainly dominant for all of these flows, the effects of buoyancy still play a role in determining the bubble size distributions 
and their variation with depth. Furthermore, it is clear that for a given flow the effects of buoyancy are greater for larger bubbles.

\section{Conclusions}

A phase Doppler anemometer (PDA) system was used to measure the air bubble size distributions downstream of two-dimensional hydraulic jumps. Air bubbles located $1 \mathrm{~m}$ downstream of the hydraulic jump toe were 1-500 $\mu \mathrm{m}$ in diameter, and the majority were approximately $40 \mu \mathrm{m}$ in diameter. Total bubble count rate and void fraction profiles indicate that there are more bubbles at intermediate elevations than near the bottom of the flume or at the free surface. It appears that most of the air bubbles are entrained at the elevation of the shear layer, $y=h_{1}$, and are dispersed from that elevation by the effects of turbulent mixing and buoyancy. At larger downstream distances, such as in the present study, the displacement by these phenomena is significant and the relative magnitude of their effects on the motion of the air bubbles seems to depend strongly on bubble size, and also on the energy of the upstream flow.

\section{Acknowledgments}

The authors are grateful to Professor Richard Skop at the University of Miami for use of his bubbling frits. They also acknowledge the technical assistance of Clifford Weisman at Dantec Measurement Technology and the financial support of the Office of Naval Research under grant number N00014-94-1-1210.

\section{Bibliography}

—. SIZEware User's Guide. Dantec Measurement Technology, 1991. 
A.F. Babb and H.C. Aus. Measurement of Air in Flowing Water. Journal of the Hydraulics Division, Proceedings of the ASCE, 107(HY12):1615-1630, 1981.

H. Chanson. Air bubble entrainment in free surface flows. Technical Report CH46/95, University of Queensland, June 1995.

H. Chanson. Air-water gas transfer at hydraulic jump with partially developed inflow. $W a-$ ter Research 29(10):2247-2254, 1995.

J. Hoyt and J. Sellin. Hydraulic Jump as "Mixing Layer". Journal of Hydraulic Engineering, 115(12): 1607-1614, 1989.

Z. Liu, K. Sato, and C.E. Brennen. Cavitation nuclei population dynamics in a water tunnel. ASME Cavitation and Multiphase Flow Forum, FED-153:119-125, 1993.

J.A. McCorquodale and A. Khalifa. Internal Flow in Hydraulic Jumps. Journal of Hydraulic Engineering 109(5), 684-701, 1983.

M. Mossa and U. Tolve. Flow Visualization in Bubbly Two-Phase Hydraulic Jump. Journal of Fluids Engineering 120:160-165, 1998.

N. Rajaratnam. The hydraulic jump as a wall jet. Journal of the Hydraulics Division, Proceedings of the ASCE, 124(HY5):107-132, 1965.

L. Qingchao and U. Drewes. Turbulence characteristics in free and forced hydraulic jumps. Journal of Hydraulic Research 32(6): 877-898, 1994. 
F.J. Resch, H.J. Leutheusser, and S. Alemu. Bubbly Two-Phase Flow in Hydraulic Jump. Journal of the Hydraulics Division, Proceedings of the ASCE, 100(HY1):137-149, 1974.

H. Rouse, T.T. Siao, S. Nagaratnam. Turbulence Characteristics of the Hydraulic Jump. Journal of the Hydraulics Division, Proceedings of the ASCE, 124(HY1), 1958.

M. Saffman. Automatic calibration of LDA measurement volume size. Applied Optics 26(13):2592-2597, 1987.

R.A. Skop, J.W. Brown, and W.G. Lindsley. Bubble Transport of Surface-Active Organic Compounds in Seawater: Initial Measurements and Results. Technical Report TR89-003, University of Miami, 1989.

G.B. Wallis. One dimensional two-phase flow. McGraw-Hill, New York, 1969.

F.M. White. Fluid Mechanics. McGraw-Hill, New York, 1986.

S. Wu and N. Rajaratnam. Transition from Hydraulic Jump to Open Channel Flow. Journal of Hydraulic Engineering, 122(9): 526-528, 1996. 